\title{
A FUNDAMENTAL PLANE OF SPIRAL STRUCTURE IN DISK GALAXIES
}

\author{
Benjamin L. Davis ${ }^{1}$, Daniel Kennefick ${ }^{1,2}$, Julia Kennefick ${ }^{1,2}$, Kyle B. Westfall ${ }^{3,4}$, Douglas W. Shields ${ }^{1,2}$, \\ Russell Flatman $^{5}$, Matthew T. Hartley ${ }^{2,6}$, Joel C. Berrier ${ }^{7}$, Thomas P. K. Martinsson ${ }^{8}$, and Rob A. Swaters ${ }^{9}$ \\ ${ }^{1}$ Arkansas Center for Space and Planetary Sciences, University of Arkansas, 346 1/2 North Arkansas Avenue, Fayetteville, AR 72701, USA; bld002@email.uark.edu \\ ${ }^{2}$ Department of Physics, University of Arkansas, 226 Physics Building, 835 West Dickson Street, Fayetteville, AR 72701, USA \\ ${ }^{3}$ Kapteyn Astronomical Institute, University of Groningen, P.O. Box 800, NL-9700 AV Groningen, The Netherlands \\ ${ }^{4}$ Institute of Cosmology and Gravitation, University of Portsmouth, Dennis Sciama Building, Burnaby Road, Portsmouth PO1 3FX, UK \\ ${ }^{5}$ School of Physics, Georgia Institute of Technology, 837 State Street, Atlanta, GA 30332, USA \\ ${ }^{6}$ Physics Department, California Institute of Technology, 1200 East California Boulevard, Pasadena, CA 91125, USA \\ ${ }^{7}$ Department of Physics and Astronomy, Rutgers, The State University of New Jersey, 136 Frelinghuysen Road, Piscataway, NJ 08854-8019, USA \\ ${ }^{8}$ Leiden Observatory, P.O. Box 9513, NL-2300 RA Leiden, The Netherlands \\ ${ }^{9}$ National Optical Astronomy Observatory, 950 North Cherry Avenue, Tucson, AZ 85719, USA \\ Received 2014 December 29; accepted 2015 March 8; published 2015 March 24
}

\begin{abstract}
Spiral structure is the most distinctive feature of disk galaxies and yet debate persists about which theory of spiral structure is correct. Many versions of the density wave theory demand that the pitch angle be uniquely determined by the distribution of mass in the bulge and disk of the galaxy. We present evidence that the tangent of the pitch angle of logarithmic spiral arms in disk galaxies correlates strongly with the density of neutral atomic hydrogen in the disk and with the central stellar bulge mass of the galaxy. These three quantities, when plotted against each other, form a planar relationship that we argue should be fundamental to our understanding of spiral structure in disk galaxies. We further argue that any successful theory of spiral structure must be able to explain this relationship.
\end{abstract}

Key words: galaxies: evolution - galaxies: fundamental parameters - galaxies: kinematics and dynamics galaxies: spiral - galaxies: structure

\section{INTRODUCTION}

Spiral structure is a commonplace and visually striking feature of many galaxies and yet there is still disagreement as to the correct theory that explains its origin after decades of debate. The first well-known theory (Lin \& Shu 1964) is that density waves propagating through the disk of the galaxy are the responsible agent. The density wave theory for spiral modes, described in detail by Bertin \& Lin (1996), calls for a long-lived, quasi-steady global spiral pattern. Others call for more transient spiral patterns, whether from swing-amplified noise (Goldreich \& Lynden-Bell 1965; Julian \& Toomre 1966), recurrent cycles of groove modes (Sellwood 2000), or superposed transient instabilities (Sellwood \& Carlberg 2014). Other theories have also been proposed, with one in particular, the manifold theory (Athanassoula et al. 2009a, 2009b, 2010), rejecting the density wave concept altogether in favor of an explanation involving stars in chaotic highly eccentric orbits.

The density wave theory, as originally articulated by Lin \& Shu (1966), had a very specific prediction for the pitch angle of the spiral pattern produced by the waves. They calculated the pitch angle to be a ratio of the density of material in the galaxy's disk to a certain quantity made up of the frequencies of orbital motions in the disks,

$$
\tan |P|=\frac{2 \pi m G\left(\sigma_{o}+F \sigma_{*}\right)}{\left(\kappa^{2}-(\omega-m \Omega)^{2}\right)},
$$

where $P$ is the logarithmic spiral arm pitch angle and $m$ is the number of spiral arms in the pattern, $G$ is the gravitational constant, $\Omega$ is the angular frequency of orbits of particles in the disk, $\kappa$ is the epicyclic frequency of the same particles, and $\omega$ is the frequency associated with the perturbation that excites the density waves. Note that as long as this perturbation is some form of self-excitation arising from within the disk itself, it follows that all of the terms in the denominator should depend on the mass of the central bulge of the galaxy. The simplest case of a dominant bulge (approximated as a point mass) would imply, for instance, $\Omega \propto \sqrt{M}$ and $\kappa \propto \sqrt{M}$, with $M$ being the central mass. The numerator depends on the density of gas in the disk, $\sigma_{o}$, and the stellar disk density, $\sigma_{*}$, with a factor $F$, called the reduction factor, which underweights the stellar density (compared to the gas density), since it is primarily within the gas that the density wave propagates. In this Letter we will present some evidence that $F<<1$.

Focusing on the masses and densities involved in this relation, we find that

$$
\tan |P| \propto \frac{\sigma_{o}+F \sigma_{*}}{M_{o}}
$$

where $M_{o}$ is the mass of the galactic bulge, or else the total mass interior to the radius in question.

This formula is known to work very well in the case of spiral density waves in Saturn's rings (Shu 1984). Bulge-dominated galaxies are not too distant from the Saturnian situation of a small dense core with negligible mass in the disk, though diskdominated galaxies are obviously far more complex. Generally speaking, the density wave theory predicts that the pitch angle of the spiral arms in galaxies does depend on the radial distribution of matter in the galaxy, and experimental studies concur (Seigar et al. 2006, 2014). Results of this type broadly agree with Lin \& Shu (1966) that a thin (dense) disk and massive (small) central bulge should result in a tight (loose) spiral. This is not surprising, since we would expect a standing wave pattern (such as in a vibrating string) to depend on the 
ratio of a restoring force or tension (in this case the central mass, or at least the mass inside a given radius $R$ ) to the density of the medium (in this case the density of gas in the disk at radius $R$ ). Although the precise nature of the relation between these three quantities can be expected to vary between galaxies of different types (bulge-dominated versus disk-dominated, for instance), we show in this Letter that the three quantities, spiral arm pitch angle, central bulge mass, and gas density in the disk, do strongly correlate to form a fundamental plane that may play a similar role in tying gross features of disk galaxies to that played by the fundamental plane of elliptical galaxies (Djorgovski \& Davis 1987; Dressler et al. 1987).

We take our sample disk galaxies from the DiskMass Survey (DMS; Bershady et al. 2010), which is ideal for our purposes since it deals with the disk densities of a sample of face-on galaxies and includes measurements of the central bulge mass. Using the technique of Davis et al. (2012), we measure the pitch angle for these galaxies and find that our sample of 24 galaxies, when plotted in a volume defined by these three quantities, delineates a plane with very low scatter. There is only a $0.0047 \%$ chance that this plane could have been formed by statistical accident.

The plane satisfies a number of requirements, which one would expect of a useful fundamental plane. The plane is steeply inclined across the volume formed by the three related quantities. In other words, it is not merely a relation between two of the three quantities, with the third essentially irrelevant. The galaxies are distributed quite widely and fairly uniformly across the plane. There is no particular evidence of a favored curvilinear relation on the face of the plane. Finally, and most importantly, the plane is oriented as one would expect on the basis of the density wave theory. A large bulge mass and a rarified disk produces the tightest spirals. A small bulge and a dense disk produce the loosest spirals. We submit that any successful theory of galactic spiral structure must be able to explain this result.

One final point is worthy of note. The DMS measured not only the density of atomic hydrogen in the disk of each galaxy (the quantity used in our relation), but also the density of molecular hydrogen and the dynamical disk mass density (the total density in the disk). Our results suggest that it is the gas density, not the total density in the disk, that matters for spiral density waves. This suggests an apparent decoupling between the stars and gas. The fact that the density of atomic hydrogen fits noticeably better than that for molecular hydrogen may simply be due to the fact that it is a much more reliable measurement, since molecular hydrogen is estimated indirectly from observations of other molecules, not hydrogen itself (Westfall et al. 2011; Martinsson et al. 2013a).

\section{DATA AND ANALYSIS}

The DMS PPak Sample (Martinsson et al. 2013b) consists of 30 nearly face-on galaxies whose disk densities have previously been closely studied. However, four of these galaxies do not have central stellar bulge masses available and three provide them only as upper limits, so they are excluded from our sample. In addition to the 23 remaining DMS galaxies, we also include our own Galaxy, the Milky Way, in our sample of galaxies.

In addition to the method described in Davis et al. (2012), which utilizes a two-dimensional fast Fourier transform software called 2DFFT, we also measured pitch angles for all of the sample galaxies using new software called Spirality. Spirality (D. W. Shields et al. 2015, in preparation) is a novel method for measuring spiral arm pitch angle by fitting galaxy images to spiral coordinate systems (templates) of known pitch. For a given pitch angle template, the mean pixel value is found along each of typically 1000 spiral axes. The fitting function, which shows a local maximum at the best-fit pitch angle, is the variance of these means. In other words, we choose the pitch angle that exhibits the greatest contrast between the mean luminosity along the spiral axes. The presumption is that where the pitch angle of the spiral axes is equal to the pitch angle of the galaxy's spiral arms there will be some axes that fall precisely along the true spiral arms (and thus are much brighter in the mean) and some that never coincide with the true spiral arms (and thus are, on average, dim). Where the pitch of the axes is not equal to the pitch of the spiral arms, each axis will cross the true spiral arms a roughly equal number of times, making the mean brightness along each axis roughly equal. Error bars are found by varying the inner radius of the measurement annulus and finding the standard deviation of the best-fit pitch angles.

The two techniques yield measurements that agree within the error bars in almost all cases. As a final and important test, we visually inspected each galaxy, comparing them to overlays of synthetic spirals on transparency paper, in order to confirm the measured pitch angle. Our overlay transparencies showed spirals of different sizes and different pitch angles in steps of $5^{\circ}$ from $5^{\circ}$ to $85^{\circ}$. We were therefore able to visually confirm the pitch angle to within $5^{\circ}$. We were satisfied in all cases that the measured pitch angle of $2 D F F T$ was reliable and strongly supported by the combination of Spirality and visual inspection. For the sake of consistency, we chose to use only the results of $2 D F F T$ in this Letter. The pitch angles, $P$, given in Table 1 are the results of the $2 D F F T$ routine.

The images used were obtained from the NASA/IPAC Extragalactic Database, ${ }^{10}$ and/or from the pODI (partial One Degree Imager) camera on the WIYN $3.5 \mathrm{~m}$ telescope. The WIYN images were all acquired as $120 \mathrm{~s}$ exposures, calibrated using QuickReduce1.0 from the ODI Pipeline, Portal, and Archive, ${ }^{11}$ and processed using a five-point dither pattern for each galaxy and subsequently stacking the images using SWarp (Bertin et al. 2002). Additionally, KPNO $2.1 \mathrm{~m}$ imaging for UGC 463, 1529, 1908, 4036, and 11318 were measured to confirm previous pitch angle measurements. Unless otherwise specified (Milky Way data have been determined in very different ways than other galaxies), all data for stellar galactic bulge masses $\left(M_{\star}^{\text {bulge }}\right)$ and maximum neutral atomic hydrogen $\left(\mathrm{H}_{\mathrm{I}}\right)$ gas mass surface densities $\left(\Sigma_{H_{I}}^{\max }\right)$ come from Martinsson (2011) and Martinsson et al. (2013a); see Table 1. For the determination of the stellar bulge masses, the $K$-band light profile was decomposed into a central Sérsic component (convolved with a seeing disk) and a number of exponential disks (Martinsson et al. 2013b). The bulge masses were determined using the integral of the light from the central Sérsic component and the mass-to-light ratio $(M / L)$ derived from the disk using vertical velocity dispersions (Martinsson et al. 2013a). Gas densities were determined from $21 \mathrm{~cm}$ line measurements (Martinsson 2011).

\footnotetext{
${ }^{10} \mathrm{http} / / /$ ned.ipac.caltech.edu/

11 http://portal.odi.iu.edu
} 


\section{RESULTS}

We find a best linear fit for Equation (2) from the included data sample of 24 galaxies of

$$
\begin{aligned}
\tan |P|= & (0.375 \pm 0.092) \frac{\sum_{H_{I}}^{\max } /\left(M_{\odot} p c^{-2}\right)}{\log \left(M_{\star}^{\text {bulge }} / M_{\odot}\right)} \\
& +(0.127 \pm 0.049) .
\end{aligned}
$$

The rms error (rmsE) is equal to 0.0909 (a residual scatter of $31.2 \%$ per galaxy on average), with $R^{2}=0.344$, and a $p$-value equal to $2.59 \times 10^{-3}$ for Equation (3). A plot of this linear fit, along with the included data sample, is given in Figure 1.
The formula describing the fundamental plane for spiral galaxies from the sample is as follows:

$$
\begin{aligned}
& \frac{\Sigma_{H_{I}}^{\max }}{M_{\odot} p c^{-2}}=(5.70 \pm 1.40) \tan |P| \\
& -(0.677 \pm 0.199) \log \left(M_{\star}^{\text {bulge }} / M_{\odot}\right)+(9.29 \pm 1.96) .
\end{aligned}
$$

$\mathrm{rmsE}=0.770 M_{\odot} p c^{-2}$ (a residual scatter of $16.7 \%$ per galaxy on average ${ }^{12}$ ), with $R^{2}=0.613$, and a $p$-value $=4.71 \times 10^{-5}$ for Equation (4). It is interesting to note that the addition of the extra dimension cuts the residual scatter approximately in half. A three-dimensional plot of this plane, along with the included

\begin{tabular}{|c|c|c|c|c|c|c|c|c|}
\hline $\begin{array}{c}\text { Galaxy Name } \\
(1) \\
\end{array}$ & $\begin{array}{l}\text { Type } \\
(2) \\
\end{array}$ & $\begin{array}{c}\text { Band } \\
(3)\end{array}$ & $\begin{array}{c}\text { Image Source } \\
(4)\end{array}$ & $\begin{array}{c}m \\
(5) \\
\end{array}$ & $\begin{array}{c}\tan |P| \\
(6) \\
\end{array}$ & $\begin{array}{c}\log \left(M_{\star}^{\text {bulge }} / M_{\odot}\right) \\
(7)\end{array}$ & $\begin{array}{c}\sum_{H_{l}}^{\max } /\left(M_{\odot} \mathrm{pc}^{-2}\right) \\
(8)\end{array}$ & $\begin{array}{c}\text { Excluded } \\
(9) \\
\end{array}$ \\
\hline Milky Way & $\mathrm{SBc}$ & $21 \mathrm{~cm}$ & 1 & 4 & $0.414 \pm 0.051^{\mathrm{a}}$ & $9.95 \pm 0.03^{b}$ & $4.98 \pm 0.53^{\mathrm{c}, \mathrm{d}}$ & $\ldots$ \\
\hline UGC 448 & $\mathrm{SABc}$ & $r$ & 2 & 4 & $0.327 \pm 0.033$ & $9.76_{-0.51}^{+0.23}$ & $4.58 \pm 0.46$ & $\cdots$ \\
\hline UGC 463 & $\mathrm{SABc}$ & $B$ & 3 & 3 & $0.412 \pm 0.066$ & $9.35_{-0.41}^{+0.21}$ & $6.18 \pm 0.66$ & $\cdots$ \\
\hline UGC 1081 & $\mathrm{SBc}$ & $r$ & 2 & 2 & $0.452 \pm 0.064$ & $8.81_{-0.24}^{+0.16}$ & $6.25 \pm 0.62$ & $\ldots$ \\
\hline UGC 1087 & $\mathrm{Sc}$ & $r$ & 2 & 2 & $0.188 \pm 0.039$ & $8.64_{-0.25}^{+0.16}$ & $4.42 \pm 0.46$ & $\ldots$ \\
\hline UGC 1529 & $\mathrm{Sc}$ & $645.0 \mathrm{~nm}^{\mathrm{e}}$ & 4 & 3 & $0.490 \pm 0.096$ & $8.98_{-0.39}^{+0.20}$ & $6.59 \pm 0.66$ & $\cdots$ \\
\hline UGC 1635 & $\mathrm{Sbc}$ & $r$ & 2 & 3 & $0.209 \pm 0.014$ & $8.74_{-0.29}^{+0.17}$ & $2.60 \pm 0.32$ & $\cdots$ \\
\hline UGC 1862 & $\mathrm{SABcd}^{1}$ & $r$ & 2 & 2 & $0.444 \pm 0.074$ & & $9.14 \pm 0.91$ & $\checkmark$ \\
\hline UGC 1908 & $\mathrm{SBc}^{2}$ & $645.0 \mathrm{~nm}^{\mathrm{e}}$ & 4 & 3 & $0.376 \pm 0.069$ & $9.68_{-0.54}^{+0.23}$ & $4.62 \pm 0.46$ & $\cdots$ \\
\hline UGC 3091 & $\mathrm{SABd}$ & $i$ & 2 & 2 & $0.555 \pm 0.092$ & & $5.59 \pm 0.56$ & $\checkmark$ \\
\hline UGC 3140 & $\mathrm{Sc}$ & $r$ & 2 & 3 & $0.290 \pm 0.090$ & $9.65_{-0.24}^{+0.15}$ & $4.87 \pm 0.54$ & $\ldots$ \\
\hline UGC 3701 & Scd & $r$ & 2 & 2 & $0.276 \pm 0.090$ & $8.69_{-0.31}^{+0.18}$ & $5.55 \pm 0.57$ & $\ldots$ \\
\hline UGC 3997 & $\operatorname{Im}$ & $g$ & 5 & 2 & $0.185 \pm 0.048$ & $8.53_{-0.27}^{+0.17}$ & $5.01 \pm 0.54$ & $\ldots$ \\
\hline UGC 4036 & SABbc & $645.0 \mathrm{~nm}^{\mathrm{e}}$ & 4 & 2 & $0.268 \pm 0.021$ & $8.92_{-0.23}^{+0.15}$ & $5.20 \pm 0.56$ & $\ldots$ \\
\hline UGC 4107 & $\mathrm{Sc}$ & $g$ & 5 & 2 & $0.371 \pm 0.041$ & $8.65_{-0.31}^{+0.18}$ & $5.42 \pm 0.54$ & $\cdots$ \\
\hline UGC 4256 & $\mathrm{SABc}$ & $g$ & 5 & 2 & $0.555 \pm 0.099$ & $9.29_{-9.29}^{+0.36}$ & $9.75 \pm 0.98$ & $\checkmark$ \\
\hline UGC 4368 & Scd & $g$ & 5 & 2 & $0.439 \pm 0.043$ & $9.21_{-0.41}^{+0.21}$ & $5.95 \pm 0.66$ & $\ldots$ \\
\hline UGC 4380 & Scd & $g$ & 5 & 3 & $0.430 \pm 0.095$ & $8.86_{-0.20}^{+0.13}$ & $4.08 \pm 0.41$ & $\ldots$ \\
\hline UGC 4458 & $\mathrm{Sa}$ & $g$ & 5 & 1 & $0.243 \pm 0.056$ & $10.67_{-0.39}^{+0.20}$ & $3.28 \pm 0.53$ & $\ldots$ \\
\hline UGC 4555 & $\mathrm{SABbc}$ & $g$ & 5 & 2 & $0.213 \pm 0.017$ & $8.96_{-0.39}^{+0.20}$ & $4.58 \pm 0.47$ & $\ldots$ \\
\hline UGC 4622 & Scd & $g$ & 5 & 4 & $0.401 \pm 0.099$ & $9.89_{-0.41}^{+0.21}$ & $3.50 \pm 0.38$ & $\cdots$ \\
\hline UGC 6903 & SBcd & $g$ & 5 & 2 & $0.283 \pm 0.041$ & $8.03_{-0.62}^{+0.25}$ & $4.94 \pm 0.59$ & $\cdots$ \\
\hline UGC 6918 & $\mathrm{SABb}^{3}$ & F606W & 6 & 3 & $0.306 \pm 0.044$ & $8.04_{-8.04}^{+0.70}$ & $7.04 \pm 0.72$ & $\checkmark$ \\
\hline UGC 7244 & SBcd & $g$ & 5 & 2 & $0.627 \pm 0.105$ & & $5.53 \pm 0.60$ & $\checkmark$ \\
\hline UGC 7917 & $\mathrm{SBbc}$ & $g$ & 5 & 3 & $0.278 \pm 0.025$ & $10.01_{-10.01}^{+00.34}$ & $2.70 \pm 0.28$ & $\checkmark$ \\
\hline UGC 8196 & $\mathrm{Sb}$ & $g$ & 5 & 5 & $0.144 \pm 0.009$ & $10.73_{-0.26}^{+0.16}$ & $2.74 \pm 0.28$ & $\cdots$ \\
\hline UGC 9177 & Scd & $g$ & 5 & 2 & $0.256 \pm 0.035$ & $9.55_{-0.58}^{+0.24}$ & $3.92 \pm 0.42$ & $\ldots$ \\
\hline UGC 9837 & $\mathrm{SABc}$ & $g$ & 5 & 6 & $0.482 \pm 0.061$ & $8.35_{-0.29}^{+0.17}$ & $7.95 \pm 0.80$ & $\cdots$ \\
\hline UGC 9965 & Sc & $g$ & 5 & 3 & $0.237 \pm 0.037$ & & $5.63 \pm 0.58$ & $\checkmark$ \\
\hline UGC 11318 & $\mathrm{SBbc}$ & $645.0 \mathrm{~nm}^{\mathrm{e}}$ & 4 & 3 & $0.569 \pm 0.101$ & $9.69_{-0.50}^{+0.23}$ & $6.51 \pm 0.67$ & $\cdots$ \\
\hline UGC 12391 & $\mathrm{SABc}$ & $r$ & 2 & 4 & $0.235 \pm 0.091$ & $8.98_{-0.28}^{+0.17}$ & $4.90 \pm 0.49$ & $\cdots$ \\
\hline
\end{tabular}
data sample, is given in Figure 2. ${ }^{13}$

Table 1

Sample

Notes. Columns: (1) Galaxy name. (2) Hubble morphological type from either the UGC (Nilson 1973) or RC3 (de Vaucouleurs et al. 1991) catalogs. Notes on morphologies: 1 = peculiar, 2 = starburst, and $3=$ AGN. (3) Filter waveband/wavelength used for pitch angle calculation. (4) Telescope/literature source of imaging used for pitch angle calculation. (5) Harmonic mode (number of spiral arms). (6) Tangent of the pitch angle of the galactic logarithmic spiral arms. (7) Base 10 logarithm of the stellar bulge mass of the galaxy, in solar masses. (8) Maximum surface density in the galactic $\mathrm{H}_{\mathrm{I}}$ gas, in solar masses per square pc. (9) Indication of galaxies that are excluded in fittings due to missing measurements or measurements that are merely upper limits. Image Sources: (1) Levine et al. (2006); (2) WIYN 3.5 m pODI; (3) JKT 1.0 m; (4) Palomar 48 inch Schmidt; (5) SDSS; (6) HST.

${ }^{a}$ Levine et al. (2006).

${ }^{\mathrm{b}}$ McMillan (2011).

${ }^{\mathrm{c}}$ No error estimates were provided by its reference so we have assigned the mean error of the included sample, $\pm 0.53 M_{\odot} p c^{-2}$.

${ }^{\mathrm{d}}$ Calculated using Equation (2) from Ferrière (2001).

e IIIaJ emulsion. 


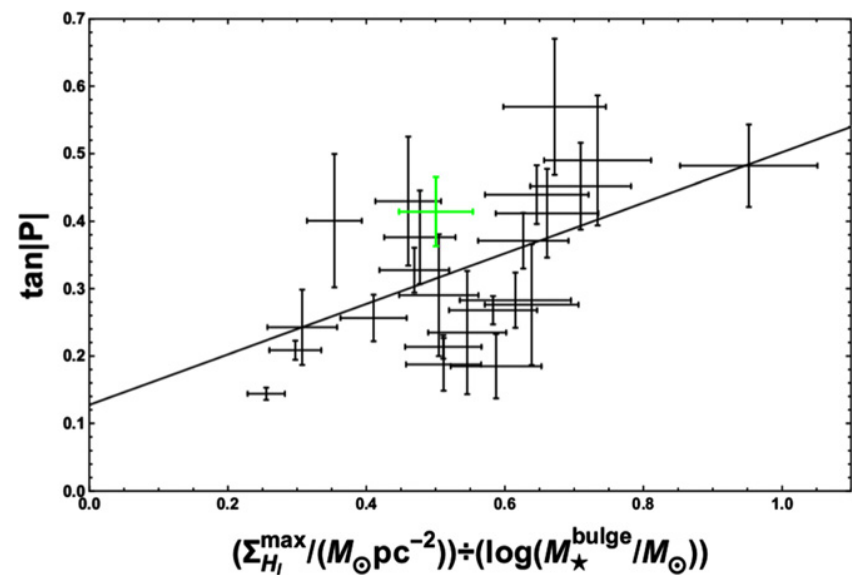

Figure 1. Two-dimensional plot of the linear fit defined by the multivariate normally distributed sampling fit of Equation (3) depicted by the solid black line, along with the plotted points of the 24 galaxy member data set. The Milky Way is depicted distinctly in green. The axes $[x, y]$ depict $[\tan |P|$, $\left.\left(\Sigma_{H_{I}}^{\max } /\left(M_{\odot} p c^{-2}\right)\right) / \log \left(M_{\star}^{\text {bulge }} / M_{\odot}\right)\right]$, respectively.

The errors presented in Equations (3) and (4) have been determined by sampling individual data points from multivariate normal distributions using the following algorithm.

1. For each measurement, draw a new measurement based on multivariate normal distributions with the mean and variance of each variable for all 24 galaxies.

2. Fit linear (or planar) best-fit coefficients to the 24 points.

3. Repeat steps 1 and $210^{6}$ times, saving the fitted coefficients after each step.

4. Use the distribution of the coefficients based on these $10^{6}$ fits to define the best-fitting (median) coefficients and their error (the $1 \sigma$ confidence interval of the distribution).

The orientation of the fundamental plane illustrated in Figure 2 is exactly as one would expect on the basis of the spiral density wave theory. According to Equation (2), the pitch angle is minimized (tightest winding) when the $\mathrm{H}_{\mathrm{I}}$ mass surface density is low and the central mass is high. Alternatively, the pitch angle is maximized (loosest winding) when the $\mathrm{H}_{\mathrm{I}}$ mass surface density is high and the central mass is low. This behavior is illustrated in both figures. Note in the middle panel of Figure 2 how the plane slopes from the lower left front (low pitch angle, low $\mathrm{H}_{\mathrm{I}}$ mass surface density, and high central mass) corner of the cube to the upper right back (high pitch angle, high $\mathrm{H}_{\mathrm{I}}$ mass surface density, and low central mass) corner of the cube. Furthermore, this indicates that the shape of the plane is strongly correlated to all three variables (the individual variable $p$-values of the intercept, $\tan |P|$, and $\log \left(M_{\star}^{\text {bulge }} / M_{\odot}\right)$ are $9.97 \times 10^{-5}, 8.03 \times 10^{-5}$, and $9.41 \times 10^{-4}$, respectively, for Equation (4)).

\section{DISCUSSION}

If one favors the standing wave picture of spiral structure, our result is not unexpected. In analogy with standing waves on a string, we would expect the wavelength of the resulting

\footnotetext{
12 Compare this to the fundamental plane for elliptical galaxies, which has a residual scatter of $\sim 20 \%$ per galaxy on average (Kormendy \& Djorgovski 1989).

13 A 3D animated gif of this figure can be accessed at http://dafix.uark.edu/ $\sim$ ben/movie.gif.
}

pattern to be strongly determined by the tension or restoring force (in this case the central gravitating mass) and the density of the medium (in this case the gas in the disk). It is worth noting that our case is probably analogous to a string with nonuniform density, since the gas density generally falls off with increasing radius in a galactic disk. ${ }^{14}$ Additionally, it seems reasonable that the gravitational restoring force increases with increasing radius, since there will be more mass inside the given radius. Both effects would tend to cause the pitch angle to tighten with increasing radius, and this effect is often seen in spiral galaxies (see, e.g., Davis et al. 2012).

It has been often proposed in the past that different mechanisms may explain spiral structure in different galaxies; for instance, the mechanism that produces grand design spirals may differ from the one that produces flocculent spirals (e.g., D'Onghia et al. 2013). Furthermore, it has been demonstrated that galaxies that appear to have grand design structure in infrared light can appear flocculent in blue images, suggesting that stellar and gaseous disks are decoupled (Grosbol \& Patsis 1998). It is striking that the sample used in this study contains quite a few flocculent or multi-armed patterns, which are not grand design. The existence of a very low scatter planar correlation for all of these galaxies is thus very significant and implies that different galactic morphologies all adhere to the same imposed mechanism of density wave theory. It is true that four of the galaxies have a noticeably greater scatter than the others, and further study with larger samples might yet support the existence of two kinds of spiral structure. It is worth noting that two of these four galaxies represent the extremes of gas density for the sample, one having clearly the highest gas density in its disk, another clearly the lowest.

In recent years, there has been some discussion that spiral arms may be quite transient, persisting for only one or two revolutions of the disk galaxy (Toomre \& Kalnajs 1991). In recent years, there have been attempts to show theoretically that more long-lasting spiral patterns are possible (Sellwood \& Carlberg 1984; D’Onghia et al. 2013). This Letter suggests that even if spiral patterns are transient, some resonant mechanism compels the pattern, when it reforms, to resume something close to its previous pitch angle.

The relation discovered here might be useful as a tool in the study of disk galaxies. One of the three quantities, disk density, is relatively difficult to measure. The relation found here could be used to measure it indirectly from the other two quantities (pitch angle and central bulge mass), which would be easier to measure. In addition, the existence of the three-way correlation may enable more careful studies of the important relation between pitch angle and central mass, which is itself a very useful marker for quantities such as the central black hole mass (Seigar et al. 2008; Berrier et al. 2013).

It has long been known that pitch angle does depend on the distribution of mass (e.g., Seigar et al. 2006, 2014) and on the size of the central bulge (for instance, the observed correlation between pitch angle and sigma reported in Seigar et al. 2008 and Berrier et al. 2013, as well as the qualitative relation of pitch angle to bulge size featured in the Hubble classification). In addition, it has been reported that pitch angle varies with the total mass of gas in galactic disks (Roberts et al. 1975). This Letter demonstrates the fundamental way in which we can

\footnotetext{
${ }^{14}$ This is true for the total gas density; however, the atomic gas density generally has a peak value at some radius and decreases toward the center due to conversion of the atomic to molecular gas.
} 

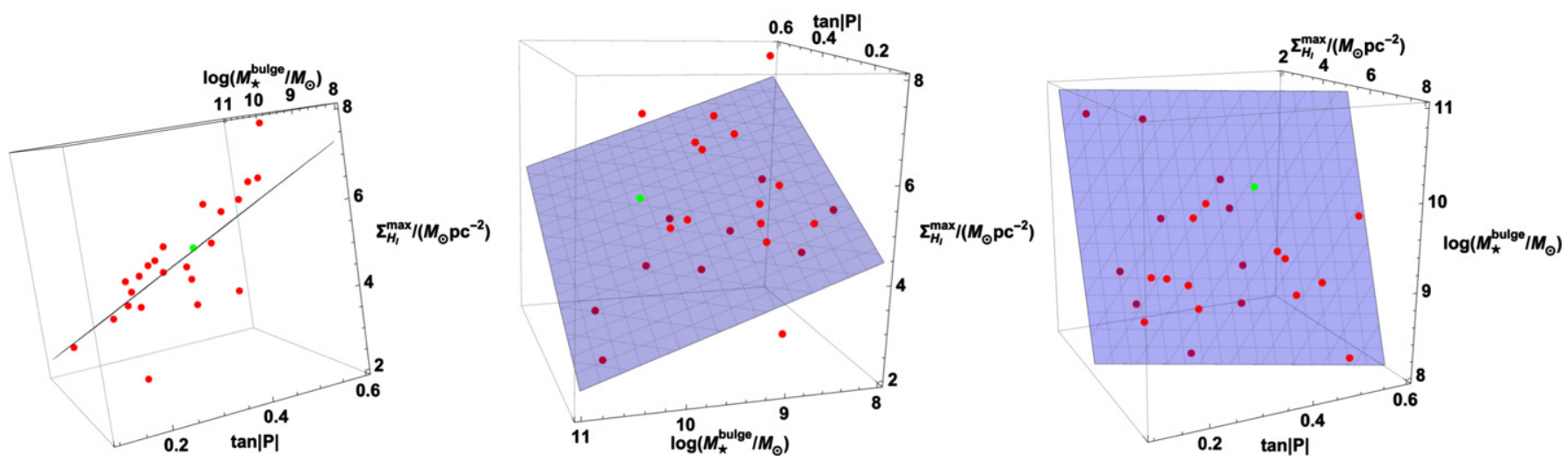

Figure 2. Three-dimensional plot of the plane defined by the fit of Equation (4) with the multivariate normally distributed sampling depicted by a translucent blue meshed surface, along with the plotted points of the 24 galaxy member included data set (depicted by red spheres with the Milky Way in green). Note that the points will appear slightly darker when they are projected behind and partially obscured by the plane. The axes $[x, y, z] \operatorname{depict}\left[\tan |P|, \log \left(M_{\star}^{\text {bulge }} / M_{\odot}\right), \Sigma_{H_{I}}^{\max }\right], \operatorname{respectively}$. Left: the view has been oriented parallel to the plane. Middle: the view has been oriented at an orientation sufficient to view the face of the plane. Right: the view has been projected along an orthogonal vector above the plane.

understand how the spiral structure depends on the distribution of mass in disk galaxies. Furthermore, it illustrates how the qualitative Hubble morphological types can exhibit varying pitch angles for galaxies that have similarly sized bulges and are thus categorized as the same type. These galaxies likely have different gas densities in their disks. Although the density wave theory provided the inspiration for this study, other theories may also be able to explain this result. Certainly, any successful theory of spiral structure must be able to do so.

The authors thank Marc Seigar and Matthew Bershady, who provided valuable comments on drafts of the Letter. We thank the National Optical Astronomy Observatory for observing time on the WIYN $3.5 \mathrm{~m}$ telescope at Kitt Peak National Observatory. We acknowledge the use of data obtained with PPak and SparsePak IFUs on the Calar Alto and WIYN $3.5 \mathrm{~m}$ telescopes, made available by the DiskMass Survey team. This research has made use of the NASA/IPAC Extragalactic Database (NED), which is operated by the Jet Propulsion Laboratory, California Institute of Technology, under contract with the National Aeronautics and Space Administration. Plots were generated using Mathematica. Numerical computations and statistical analyses were performed with Matlab. We also thank the National Science Foundation (NSF) for REU Site grant No. 0851150, which contributed to a significant part of the data collection for this Letter. This research has made use of NASA's Astrophysics Data System. K.B.W. acknowledges support from NSF (USA) grant OISE-0754437 and NWO (NL) grant 614.000.807.

\section{REFERENCES}

Athanassoula, E., Romero-Gómez, M., Bosma, A., \& Masdemont, J. J. 2009a, MNRAS, 400, 1706

Athanassoula, E., Romero-Gómez, M., Bosma, A., \& Masdemont, J. J. 2010, MNRAS, 407, 1433

Athanassoula, E., Romero-Gómez, M., \& Masdemont, J. J. 2009b, MNRAS, 394, 67

Berrier, J. C., Davis, B. L., Kennefick, D., et al. 2013, ApJ, 769, 132
Bershady, M. A., Verheijen, M. A. W., Swaters, R. A., et al. 2010, ApJ, 716, 198

Bertin, G., \& Lin, C. C. 1996, Spiral Structure in Galaxies a Density Wave Theory (Cambridge, MA: MIT Press)

Bertin, E., Mellier, Y., Radovich, M., et al. 2002, in ASP Conf. Ser. 281, The TERAPIX Pipeline, ed. D. A. Bohlender, D. Durand, \& T. H. Handley (San Francisco, CA: ASP), 228

Davis, B. L., Berrier, J. C., Shields, D. W., et al. 2012, ApJS, 199, 33

de Vaucouleurs, G., de Vaucouleurs, A., Corwin, H. G., Jr, et al. 1991, Third Reference Catalogue of Bright Galaxies, ed. N. G. Roman et al. (Berlin: Springer)

Djorgovski, S., \& Davis, M. 1987, ApJ, 313, 59

D’Onghia, E., Vogelsberger, M., \& Hernquist, L. 2013, ApJ, 766, 34

Dressler, A., Lynden-Bell, D., Burstein, D., et al. 1987, ApJ, 313, 42

Ferrière, K. M. 2001, RvMP, 73, 1031

Goldreich, P., \& Lynden-Bell, D. 1965, MNRAS, 130, 125

Grosbol, P. J., \& Patsis, P. A. 1998, A\&A, 336, 840

Julian, W. H., \& Toomre, A. 1966, ApJ, 146, 810

Kormendy, J., \& Djorgovski, S. 1989, ARA\&A, 27, 235

Levine, E. S., Blitz, L., \& Heiles, C. 2006, Sci, 312, 1773

Lin, C. C., \& Shu, F. H. 1964, ApJ, 140, 646

Lin, C. C., \& Shu, F. H. 1966, PNAS, 55, 229

Martinsson, T. P. K. 2011, PhD thesis, Univ. Groningen

Martinsson, T. P. K., Verheijen, M. A. W., Westfall, K. B., et al. 2013a, A\&A, 557, A131

Martinsson, T. P. K., Verheijen, M. A. W., Westfall, K. B., et al. 2013b, A\&A, 557, A130

McMillan, P. J. 2011, MNRAS, 414, 2446

Nilson, P. 1973, Uppsala General Catalogue of Galaxies (Uppsala: Astronomiska Observatorium)

Roberts, M. S. 1975, in Radio Observations of Neutral Hydrogen in Galaxies, ed. A. Sandage, M. Sandage, \& J. Kristian (Chicago, IL: Univ. Chicago Press), 309

Seigar, M. S., Bullock, J. S., Barth, A. J., \& Ho, L. C. 2006, ApJ, 645, 1012 Seigar, M. S., Davis, B. L., Berrier, J., \& Kennefick, D. 2014, ApJ, 795, 90

Seigar, M. S., Kennefick, D., Kennefick, J., \& Lacy, C. H. S. 2008, ApJL, 678, L93

Sellwood, J. A. 2000, Ap\&SS, 272, 31

Sellwood, J. A., \& Carlberg, R. G. 1984, ApJ, 282, 61

Sellwood, J. A., \& Carlberg, R. G. 2014, ApJ, 785, 137

Shu, F. H. 1984, in IAU Colloq. 75, Planetary Rings, ed. R. Greenberg, \& A. Brahic (Tuscon, AZ: Univ. Arizona Press), 513

Toomre, A., \& Kalnajs, A. J. 1991, in Dynamics of Disc Galaxies, ed. B. Sundelius (Göteberg: Göteberg Univ.), 341

Westfall, K. B., Bershady, M. A., Verheijen, M. A. W., et al. 2011, ApJ, 742,18 